\title{
Primary Care Attributes Associated with Receipt of Preventive Care Services: A National Study
}

\author{
Emily C. White VanGompel, MD, MPH, Anthony F. Jerant, MD, \\ and Peter M. Franks, MD
}

Background: Primary care attributes (PCAs) encompassed by patient-centered medical homes may increase receipt of preventive care, though national studies are lacking.

Methods: We performed cross-sectional adjusted analyses of self-report data from adults in the 2007 to 2010 US Medical Expenditure Panel Surveys $(\mathrm{N}=50,457)$. PCAs were considered individually and as a total score for each respondent and included comprehensiveness (a usual source of care for new and ongoing problems, preventive care, and referrals); patient-centeredness (shared decision making); and enhanced access (night and weekend hours). Preventive care measures included mammography, influenza vaccination, annual exams, colorectal cancer screening, and Papanicolaou, prostate-specific antigen, and cholesterol testing.

Results: The total PCA score was positively associated with increased receipt of each preventive care measure. Colorectal cancer screening (18.5\%) and prostate-specific antigen testing $(20.7 \%)$ showed the largest increases across PCA score quartiles. Individual primary care attributes except enhanced access were positively associated with each preventive care measure. Enhanced access was negatively associated with annual examination (adjusted odds ratio, $0.83 ; 95 \%$ confidence interval, $0.77-0.91$ ).

Conclusion: In a nationally representative sample, greater reported exposure to key primary care attributes, with the exception of enhanced access, was associated with increased preventive care. These findings may inform best practices for maximizing preventive care delivery. ( $\mathrm{J}$ Am Board Fam Med 2015; 28:733-741.)

Keywords: Medical Home, Preventive Medicine

In national studies, greater patient-reported access to core primary care attributes, ${ }^{1,2}$ including comprehensiveness and patient-centeredness, is associated with reduced mortality. ${ }^{3}$ These attributes are now encompassed in the joint principles of the patient-centered medical home (PCMH) ${ }^{4}$ The joint principles also add further attributes to the definition of the PCMH, including enhanced ac-

This article was externally peer reviewed.

Submitted 4 March 2015; revised 23 June 2015; accepted 7 July 2015.

From the Department of Family and Community Medicine, Center for Healthcare Policy and Research, University of California Davis School of Medicine, Sacramento.

Funding: Support for this research was provided by the Agency for Healthcare Research and Quality (AHRQ) through the Quality, Safety, and Comparative Effectiveness Research Training (QSCERT) Fellowship Program (grant no. T32HS022236 to ECWV).

Conflict of interest: none declared.

Corresponding author: Emily C. White VanGompel, MD, MPH, 4860 Y St, Ste 2300, Sacramento, CA 95817 (E-mail: emily.whitevg@gmail.com). cess to care (eg, availability of evening and weekend office hours), which has previously been associated with less emergency department use and fewer total health care expenditures. ${ }^{5,6}$ Despite the emphasis placed on comprehensiveness, patient-centeredness, and extended access in prevailing health care redesign efforts, how these attributes influence utilization of preventive services at the national level is little studied. Existing research consists of ecological studies, ${ }^{7}$ small pilot projects, ${ }^{8}$ and larger comparative studies. ${ }^{9,10}$ However, nearly all have been limited to a relatively narrow group of patients (eg, those with certain chronic diseases, geriatric patients, pediatric patients) and/or to a single geographic site (eg, managed care organizations, hospital regions, single primary care practices). This is a key research gap, given that optimizing preventive care is a common goal among primary care providers, patients, and policymakers. ${ }^{1-13}$ 
Only 1 prior national study has explored whether patient access to such primary care attributes is associated with the receipt of preventive care services. ${ }^{14}$ Using data from the 2005 Medical Expenditure Panel Survey (MEPS), the authors examined how a cluster of primary care attributes, including measures reflecting comprehensiveness, patient-centeredness, and enhanced access, was globally associated with the utilization of several types of preventive care (cholesterol testing, blood pressure measurement, mammography, and prostate-specific antigen [PSA] testing). The study found that having a usual source of care (USOC) that featured the aforementioned primary care attributes was associated with higher rates of preventive services delivery. The study did not examine the independent associations of the individual primary care attributes with the various preventive care outcomes considered. Thus, it is unclear whether each of the components in the cluster contributed to increased preventive care or, if so, how strongly each attribute influenced preventive care. Studying the associations of individual core primary care attributes with the delivery of a range of common preventive services at a national level could help to identify the specific primary care attributes that are most useful to target as part of ongoing practice redesign efforts to improve preventive services delivery.

Using data from the 2007 to 2010 national MEPS, the associations of patient-reported access to selected primary care attributes-comprehensiveness, patient-centeredness, and enhanced access-with receipt of an array of preventive care services (colorectal cancer [CRC] screening; Papanicolaou, mammography, cholesterol, and PSA testing; influenza vaccination; and routine annual exams [ie, preventive care or "checkup" visits]) were examined. Based on prior research, ${ }^{10,15,16}$ we hypothesized that greater patient-reported exposure to the studied primary care attributes, whether considered as a cluster (collectively) or individually, would be associated with increased utilization of each of the studied preventive services.

\section{Methods}

We used data from the MEPS Household Component (HC) survey data sets. The MEPS is an annual national survey of health care cost and utilization in the United States, which collects survey data over a 2-year period from each individual. The HC includes items detailing sociodemographics, geographical information, health insurance, health utilization, and health care expenditures in addition to questions regarding health conditions and health status. We weighted the cluster-based sample data set to approximate a nationally representative sample of the United States. Included in this study were all respondents aged 18 and older from years 2007 to 2010 ; response rates were $56.9 \%, 59.3 \%, 57.2 \%$, and $53.5 \%$, respectively, for the survey years included.

\section{Measures}

\section{Primary Care Attributes}

We identified primary care attributes from participants' responses to individual items included in the MEPS HC questionnaire. Respondents who denied having a USOC were assigned a PCA score of 0 , and no further attributes were credited. For respondents indicating a USOC, we examined their answers to questionnaire items reflecting 3 core attributes of primary care: comprehensiveness, patient-centeredness, and enhanced access. Comprehensiveness of care was determined by 4 yes (1 point)/no (0 points) items, asking whether they visited the USOC for (1) preventive services, (2) new health problems, (3) ongoing care, and (4) referrals. Patient-centeredness of care was assessed with a single item, using a 4-point Likert response scale $(0=$ never, $0.33=$ sometimes, $0.66=$ usually, $1=$ always), asking whether the USOC invites the patient to help in determining care. Enhanced access to care was assessed with a single yes (1 point)/no (0 points) item, asking whether the USOC offers night or weekend hours. A total mean primary care attribute score was calculated (scaled to a range of 0 to 1 ) by summing responses to each answered question and dividing by the number of nonmissing questions, thus minimizing error based on missing data from any one primary care attribute question. Figure 1 shows the algorithm used to calculate the total mean primary care attribute score.

\section{Preventive Care Measures}

Preventive care measures identified from the MEPS HC included mammography, Papanicolaou, cholesterol, and PSA testing; CRC screening; influenza immunization; and routine annual exams. Criteria for adherence was, whenever possible, based on 
Figure 1. Calculation of the total primary care attributes score. PCA, primary care attribute; USOC, usual source of care.

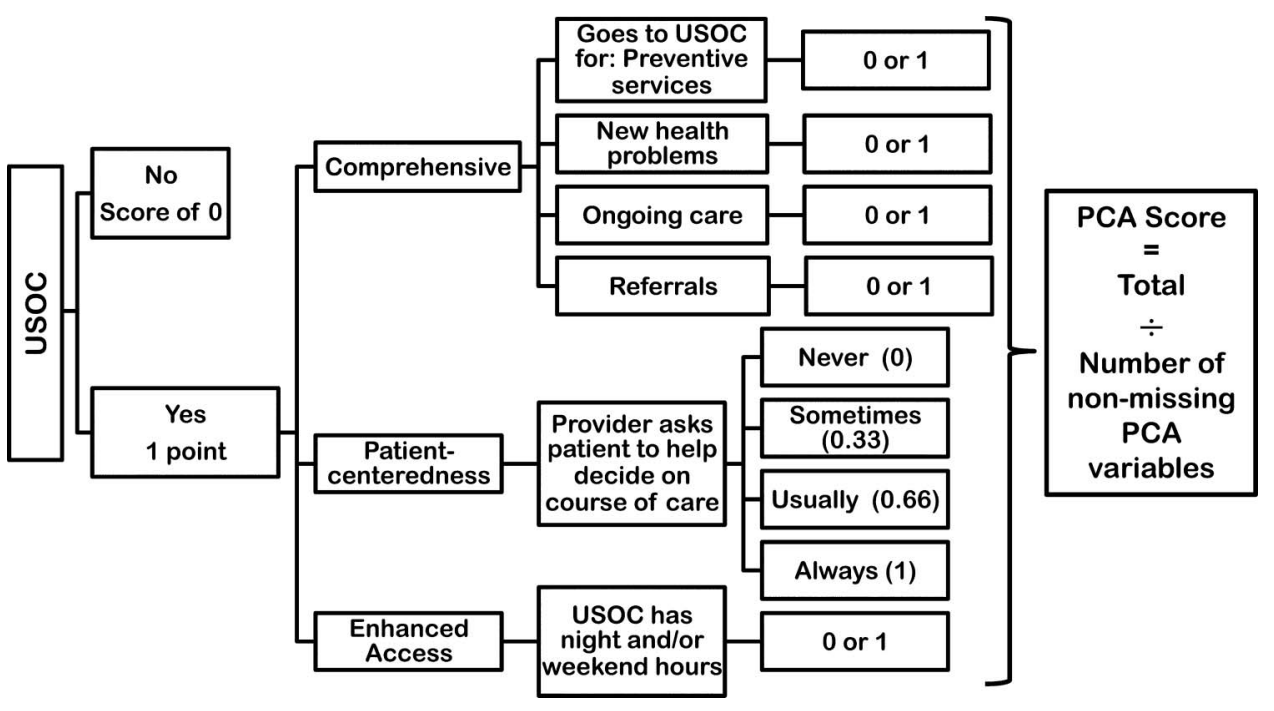

recommendations from the US Preventive Services Task Force and the Centers for Disease Control and Prevention Advisory Committee on Immunization Practices that were in existence during the study period. ${ }^{17-21} \mathrm{We}$ used common practice when guidelines were not available or at odds with common practice. $^{22,23}$ We included mammography data for female respondents aged $\geq 40$ years and defined adberent as reporting a mammogram within the past 2 years for those $\geq 50$ years and ever reporting a mammogram for those 40 to 49 years old. Papanicolaou testing was included for female respondents aged 21 to 65 years old and was defined as adherent if performed within the past 3 years. Cholesterol testing was included for all respondents aged $\geq 35$ years and defined as compliant if performed within the past year. CRC screening was included for all respondents aged $\geq 50$ years. This combined item used respondents' answers to questions about fecal occult blood testing, sigmoidoscopy, and colonoscopy. For the years 2007 to 2008 , we defined adberent as an answer of "yes" to whether the respondent had ever had a colonoscopy and/or sigmoidoscopy. Because of changes to the questionnaire after 2008, for the years 2009 to 2010 we defined adberent as having had a colonoscopy in the past 10 years, a sigmoidoscopy in the past 5 years, or a fecal occult blood test in the past 1 year. PSA data were included for all male respondents aged $\geq 50$ years and were defined as adherent if the respondent reported testing in the past 2 years. We included annual examination data for all respondents aged $\geq 18$ years and defined compliance as having had an examination within the last year.

\section{Sociodemograpbic Variables}

The following sociodemographic variables were included: age (years); sex; race/ethnicity (white, black, Hispanic, or other); insurance status (any private, only public, or uninsured); education level (none, elementary only, some high school, completed high school, some college, completed college); family income as a percentage of the federal poverty level ( $<100 \%, 100 \%$ to $124 \%, 125 \%$ to $199 \%, 200 \%$ to $399 \%$, or $\geq 400 \%$ ); and geographic region (North, Midwest, South, West).

\section{Health-Related Variables}

Five additional health-related variables were included in the analysis to control for other factors that may affect preventive care. ${ }^{24-26}$ Skepticism regarding medical care was measured with a previously validated ${ }^{27}$ scale based on Likert-type responses to 4 items (scores ranged from 1 to 5): "do not need health insurance," "health insurance not worth cost," "more likely to take risks than the average person," and "can overcome ills without medical help" (the Cronbach $\alpha$ in this data set was 0.67). We controlled for health behavior with 3 separate measures: current smoking status (smokers $=1$, others $=0$ ); seatbelt use ("always" or "nearly always" scored 0, all others scored 1); and whether the respondent was obese (body mass in$\operatorname{dex} \geq 30 \mathrm{~kg} / \mathrm{m}^{2}=1$, others $=0$ ). We measured 
disease burden as a count of 13 chronic conditions (score range, 0 to 12): hypertension, coronary heart disease, angina, heart attack, other heart disease, stroke, emphysema, chronic bronchitis, high cholesterol, cancer, diabetes, arthritis, and asthma. High cholesterol was not included as a chronic disease in analyses of cholesterol testing. Scores of the 12-item Short Form Mental Component Summary and Physical Component Summary were used to measure mental and physical health status. ${ }^{28}$

\section{Data Analysis}

Data were analyzed using Stata version 12.1 (StataCorp, College Station, TX). We used longitudinal strata, primary sampling unit identifiers, and survey weights to account for the nonrandom sampling technique in the MEPS.

Logistic regression models were used to examine the association between the key independent variable (total primary care attribute score, a continuous variable) and adherence (or not) to each preventive care measure (dependent variable). In addition, we performed a separate analysis of each individual primary care attribute measure and its association with each preventive care measure using logistic regression. We adjusted analyses for panel year, sociodemographic variables, and health-related variables. We then used the primary care attribute score as a grouped ordinal variable, using cut points based on sample size and distribution of the number of primary care attributes to examine effects on preventive care measures.

\section{Results}

A total of 68,829 respondents participated in the 2007 to 2010 MEPS; data for the primary care attributes of interest were available for 50,457 (94.5\%). Respondents with missing primary care attribute items were as follows: usual source of care, 2754 (5.5\%); ongoing source of care, $33(0.1 \%)$; preventive care, $31(0.1 \%)$; care for referrals, 45 (0.1\%); night and weekend hours, $2629(5.2 \%)$; and patient-centeredness, 1965 (3.9\%).

Tables 1 and 2 summarize the sample characteristics by primary care attribute score. The sample had an overall mean attribute score of 0.66 . Respondents who were female, white, had some form of insurance (public or private), had completed college, had a higher income, or lived in the Northeast had higher mean scores.
Table 3 shows the adjusted associations between primary care attribute score and preventive care measures. The primary care attribute score was positively associated with compliance with each preventive care measure; strength of association was strongest for PSA testing and weakest for influenza vaccination.

Table 4 shows the adjusted associations of the individual primary care attributes and the study preventive care measures. Most of the study primary care attributes were positively associated with some or all the preventive care measures. Exceptions were that having a regular provider of ongoing care for problems had no association with $\mathrm{Pa}$ panicolaou testing; patient-centeredness had no association with CRC screening; and night and weekend hours were not significantly associated with any of the preventive care measures except for having an annual examination, for which there was a significant negative association. Having a regular provider for preventive care needs, referrals, and new problems were positively associated with all the preventive care measures examined.

Table 5 shows compliance with each preventive care measure based on primary care attribute score quartile. Compliance prevalence was highest in the second highest quartile, without further increase above this. We noted the largest increase in compliance from lowest to highest quartile for CRC and PSA screening, whereas the smallest increase was noted for Papanicolaou testing.

\section{Discussion}

In a large national sample, higher patient-reported exposure to selected primary care attributes was associated with increased utilization of an array of preventive care services. The associations persisted after adjusting for multiple likely confounders, including sociodemographics, medical skepticism, health behaviors, and comorbidity. Analysis by primary care attribute component showed consistently positive associations between preventive care measures and having a regular provider for prevention, referrals, and new problems-measures of comprehensive care. Overall, these findings suggest that greater exposure to primary care attributes now encompassed by the PCMH is associated with increased preventive care utilization.

Notably departing from this general pattern were the findings regarding extended access, mea- 
Table 1. Sample Characteristics by Primary Care Attribute Score

\begin{tabular}{|c|c|c|c|}
\hline & No. & Proportion & $\begin{array}{l}\text { PCA Score } \\
\quad(\mathrm{SE})\end{array}$ \\
\hline Total & 50,457 & $100 \%$ & $0.66(0.004)$ \\
\hline \multicolumn{4}{|l|}{ Age (years) } \\
\hline $18-44$ & 24,991 & $46.71 \%$ & $0.57(0.006)$ \\
\hline $45-54$ & 9,096 & $18.57 \%$ & $0.69(0.006)$ \\
\hline $55-64$ & 7,297 & $16.18 \%$ & $0.74(0.005)$ \\
\hline$\geq 65$ & 8,019 & $18.54 \%$ & $0.80(0.004)$ \\
\hline \multicolumn{4}{|l|}{ Sex } \\
\hline Male & 23,056 & $48.49 \%$ & $0.62(0.005)$ \\
\hline Female & 26,347 & $51.51 \%$ & $0.71(0.004)$ \\
\hline \multicolumn{4}{|l|}{ Race } \\
\hline White & 23,161 & $67.84 \%$ & $0.70(0.005)$ \\
\hline Black & 9,378 & $11.58 \%$ & $0.62(0.007)$ \\
\hline Hispanic & 12,340 & $13.95 \%$ & $0.51(0.009)$ \\
\hline Other & 4,524 & $6.64 \%$ & $0.63(0.013)$ \\
\hline \multicolumn{4}{|l|}{ Insurance } \\
\hline Private, any & 10,507 & $15.39 \%$ & $0.71(0.004)$ \\
\hline Public only & 28,636 & $67.42 \%$ & $0.73(0.006)$ \\
\hline Uninsured & 10,260 & $17.19 \%$ & $0.37(0.009)$ \\
\hline \multicolumn{4}{|l|}{ Education level } \\
\hline None & 273 & $3.00 \%$ & $0.49(0.024)$ \\
\hline Elementary only & 4,294 & $5.53 \%$ & $0.59(0.012)$ \\
\hline Some HS & 7,872 & $13.04 \%$ & $0.62(0.007)$ \\
\hline Completed HS & 14,953 & $2.99 \%$ & $0.65(0.006)$ \\
\hline Some college & 10,645 & $23.97 \%$ & $0.68(0.006)$ \\
\hline Completed college & 10,570 & $27.27 \%$ & $0.71(0.006)$ \\
\hline \multicolumn{4}{|l|}{ Income } \\
\hline Poor/negative & 8,864 & $12.40 \%$ & $0.56(0.007)$ \\
\hline Near poor & 2,904 & $4.31 \%$ & $0.60(0.012)$ \\
\hline Low income & 8,086 & $13.69 \%$ & $0.60(0.009)$ \\
\hline Middle income & 15,234 & $30.54 \%$ & $0.66(0.007)$ \\
\hline High income & 14,315 & $39.05 \%$ & $0.73(0.005)$ \\
\hline \multicolumn{4}{|l|}{ Region } \\
\hline North & 7,615 & $18.42 \%$ & $0.75(0.008)$ \\
\hline Midwest & 9,844 & $21.89 \%$ & $0.71(0.009)$ \\
\hline South & 18,473 & $36.53 \%$ & $0.61(0.008)$ \\
\hline West & 12,994 & $23.17 \%$ & $0.64(0.008)$ \\
\hline \multicolumn{4}{|l|}{ Panel } \\
\hline 2007 & 10,905 & $19.52 \%$ & $0.68(0.006)$ \\
\hline 2008 & 14,946 & $30.99 \%$ & $0.65(0.007)$ \\
\hline 2009 & 13,254 & $24.63 \%$ & $0.66(0.007)$ \\
\hline 2010 & 11,352 & $24.87 \%$ & $0.67(0.006)$ \\
\hline \multicolumn{4}{|l|}{ Health-related variables } \\
\hline \multicolumn{4}{|l|}{ Smoking status } \\
\hline Nonsmoker & 34,503 & $80.75 \%$ & $0.68(0.005)$ \\
\hline Current smoker & 8,031 & $19.25 \%$ & $0.59(0.007)$ \\
\hline \multicolumn{4}{|l|}{ Seatbelt use } \\
\hline $\begin{array}{l}\text { Always or almost } \\
\text { always }\end{array}$ & 45,237 & $93.63 \%$ & $0.67(0.004)$ \\
\hline
\end{tabular}

Continued
Table 1. Continued

\begin{tabular}{lrrc}
\hline & No. & Proportion & $\begin{array}{c}\text { PCA Score } \\
\text { (SE) }\end{array}$ \\
\hline Less frequently & 2,934 & $6.37 \%$ & $0.60(0.012)$ \\
Obesity & & & \\
BMI $<30 \mathrm{~kg} / \mathrm{m}^{2}$ & 32,867 & $71.04 \%$ & $0.65(0.005)$ \\
BMI $\geq 30 \mathrm{~kg} / \mathrm{m}^{2}$ & 13,957 & $28.96 \%$ & $0.70(0.005)$ \\
Chronic health & & & \\
$\quad$ conditions (n) & & & \\
$0 \quad 22,973$ & $42.83 \%$ & $0.56(0.007)$ \\
1 & 10,270 & $21.44 \%$ & $0.67(0.005)$ \\
2 & 6,311 & $13.97 \%$ & $0.76(0.006)$ \\
3 & 4,227 & $9.21 \%$ & $0.79(0.005)$ \\
$\geq 4$ & 5,622 & $12.56 \%$ & $0.82(0.004)$ \\
\hline
\end{tabular}

*Total number of primary care attribute (PCA) variables endorsed by the respondent divided by total nonmissing PCA variables. Scores ranged between 0 and 1 , with a population mean of 0.66 .

HS, high school; SE, standard error; BMI, body mass index.

sured as the availability of evening and weekend hours, which was negatively associated with receipt of an annual examination and not significantly associated with any of the other preventive care services. This finding may reflect that visits during nontraditional office hours are more likely to focus on urgent or acute issues. Such a focus might preempt preventive care, particularly more time-consuming preventive activities such as performing a Papanicolaou test or discussing CRC screening options or the pros and cons of PSA testing. ${ }^{29,30}$ In addition, patients who value extended access may be more likely to seek out practices that offer it and to recognize (and report) that it is available, and such individuals may place a lower premium on prevention. While extended office hours have been associated with lower overall health care costs and fewer emergency department visits, ${ }^{5,6}$ these findings underscore the need for further investigation of their impact on care outcomes, including preventive care.

Table 2. Sample Characteristics for Continuous Health-related Variables

\begin{tabular}{lcc}
\hline & No. & Mean (SE) \\
\hline Mental health (MCS-12) & 45,722 & $50.9(0.1)$ \\
Physical health (PCS-12) & 45,712 & $49.5(0.1)$ \\
Medical skepticism & 45,421 & $2.1(0.01)$ \\
\hline
\end{tabular}

MCS-12, 12-item Short Form Mental Component Summary; PCS-12, 12-item Short Form Physical Component Summary; SE, standard error. 
Table 3. Adjusted Associations of the Primary Care Attribute Score with Preventive Care Adherence*

\begin{tabular}{ll}
\hline & AOR (95\% CI) \\
\hline Mammography & $2.46(2.13-2.84)$ \\
CRC screen & $2.74(2.35-3.19)$ \\
Papanicolaou test & $2.09(1.78-2.45)$ \\
Influenza vaccination & $1.84(1.69-2.01)$ \\
Annual exam & $2.38(2.21-2.58)$ \\
Cholesterol test $^{\dagger}$ & $3.55(3.07-4.10)$ \\
PSA test & $3.28(2.71-3.98)$ \\
\hline
\end{tabular}

*Adjustors: demographics, medical skepticism, health behaviors, disease burden, and mental and physical health status as measured by the 12-item Short Form Mental Component Summary and Physical Component Summary scores.

${ }^{\dagger} \mathrm{A}$ diagnosis of high cholesterol was excluded from this measure's disease burden control variable.

AOR, adjusted odds ratio; CI, confidence interval; CRC, colorectal; PSA, prostate-specific antigen.

The analysis of primary care attributes score by quartiles showed a gradient of respondents reporting prevention compliance from lowest to highest quartiles. There was little difference between the highest and second highest quartiles, suggesting a ceiling effect of a certain level of exposure to primary care attribute components. Alternatively, this could reflect the lack of increased preventive care benefit seen with the addition of night and weekend hours. Overall, the largest increase across quartiles occurred for CRC screening and PSA testing, both of which usually require a more detailed patientprovider interaction than items such as influenza vaccination or mammography. Though PSA was graded as an "I" recommendation by the US Preventive Services Task Force at the time of study data collection, it was increasingly considered the standard of care at the turn of the century. ${ }^{23}$ Interestingly, the individual analysis of patient-centeredness failed to show an association with CRC screening, which may indicate that, with counseling, patients feel they can opt out of this screening. Such an interpretation is supported by prior research regarding the effects of shared decision making on patient cancer screening decisions. ${ }^{31,32}$

Papanicolaou testing and influenza vaccination had the weakest strength of association across individual primary care attributes. Both Papanicolaou testing and the influenza vaccine are readily available in the United States through non-USOC providers (eg, Planned Parenthood for Papanicolaou testing, pharmacies or public health departments

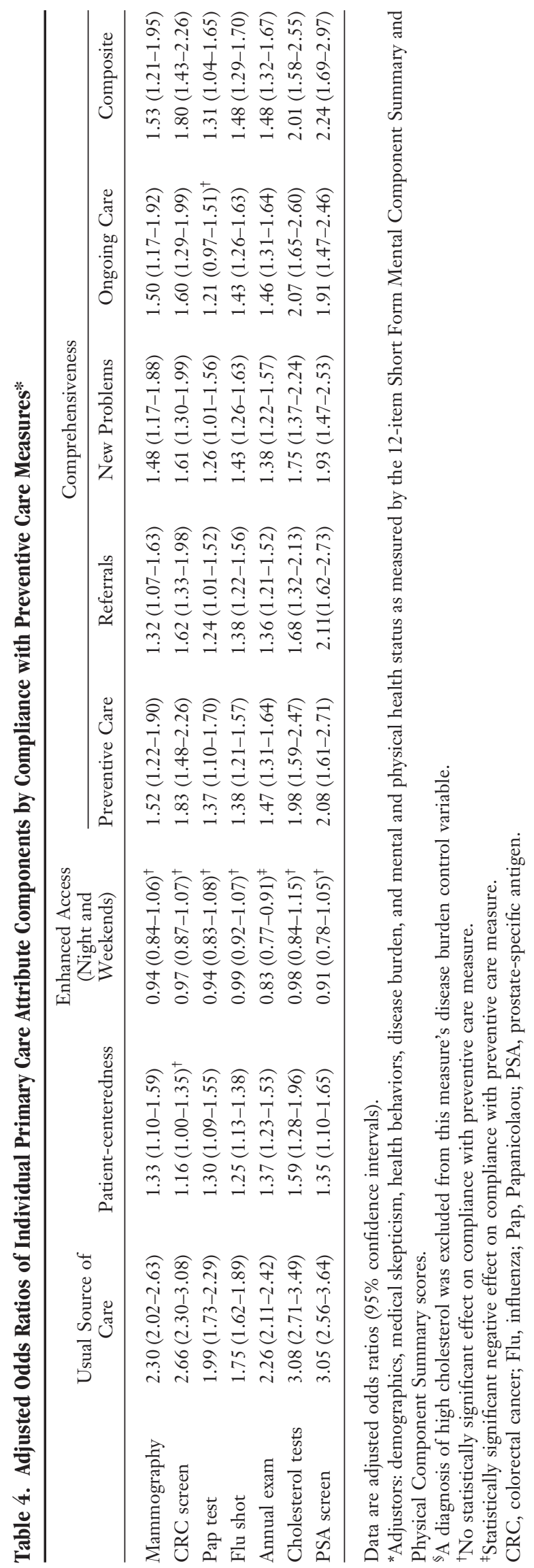


Table 5. Percentage of Respondents Adherent to Preventive Care Measures by Primary Care Attribute Score Quartile*

\begin{tabular}{lccrr}
\hline & \multicolumn{4}{c}{ Quartile } \\
\cline { 2 - 5 } & Lowest $(0-0.49)$ & Second Lowest $(0.5-0.79)$ & Second Highest (0.8-0.89) & Highest ( $\geq 0.9)$ \\
\hline Mammography & $62.5(60-65)$ & $75.5(73-78)$ & $78.9(78-80)$ & $76.9(75-78)$ \\
CRC screening & $40.6(38-43)$ & $58.7(55-62)$ & $60.9(59-62)$ & $59.1(57-61)$ \\
Papanicolaou test & $78.4(77-80)$ & $85.3(83-87)$ & $87.3(86-88)$ & $86.3(85-88)$ \\
Flu vaccination & $29.2(28-31)$ & $37.8(36-40)$ & $40.4(39-41)$ & $39.1(38-40)$ \\
Annual exam & $51.5(50-53)$ & $64.6(62-67)$ & $69.2(68-70)$ & $65.4(64-67)$ \\
Cholesterol test $^{\dagger}$ & $82.3(81-84)$ & $91.2(90-93)$ & $92.7(92-94)$ & $92.0(91-93)$ \\
PSA test & $45.5(42-49)$ & $65.9(62-70)$ & $69.6(68-71)$ & $66.2(64-69)$ \\
\hline
\end{tabular}

Data are percentages (95\% confidence intervals).

*Adjustors: demographics, medical skepticism, health behaviors, disease burden, and mental and physical health status as measured by the 12-item Short Form Mental Component Summary and Physical Component Summary scores.

${ }^{\dagger}$ A diagnosis of high cholesterol was excluded from this measure's disease burden control variable.

$\mathrm{CRC}$, colorectal cancer; Flu, influenza; PSA, prostate-specific antigen.

for the influenza vaccination). Thus these measures of prevention are likely to be less dependent on an individual having a USOC that provides these services.

This study had limitations. Most important, it does not allow us to infer causal direction, as patients who place a higher value on preventive care are possibly more likely to seek out this type of care, beyond effects captured by the medical skepticism measure. There was potential for selfreport bias: Patients who have a tendency to use preventive services may also be more likely to report exposure to the primary care attributes studied, independent of actual exposure. In addition, only selected primary care attributes are assessed in the MEPS. Other data sources are required to study additional attributes encompassed by the PCMH. Though some data were missing for the key predictor variables in our analyses, the amount of missing data was small, suggesting this would be unlikely to threaten the generalizability of our findings. In addition, the MEPS design does not allow for distinguishing between receiving testing for primary prevention or for other purposes (ie, known disease monitoring). The most problematic prevention variable in this regard is likely to be cholesterol testing, whereas others, such as influenza vaccination, are more purely preventive in nature. In addition, for CRC screening, given the long upto-date period following endoscopic screening, some individuals reporting up-to-date status in our study may have undergone screening well before their period of MEPS participation. For these respondents, the attributes of the USOC may have changed in the interval between screening and MEPS participation. Nonresponse error to the MEPS may limit the generalizability of these findings to nonresponders; however, we believe that this is the most representative sample currently available to study these associations. Our study was not designed to measure any actual health benefits of receipt of preventive care, and since the study period includes recommendations for screening that were standard of care at the time, but are now considered of questionable health benefit (annual exams and PSA testing), it is important not to equate utilization with derivation of benefit.

\section{Conclusion}

In a national sample, greater patient-reported exposure to key primary care attributes subsumed within the PCMH definition-comprehensiveness and patient-centeredness-was associated with increased receipt of preventive care. However, these attributes varied in how strongly they were associated with each of the preventive services examined. Further, one primary care attribute studied-access to night and weekend hours-was negatively associated with receipt of annual exams. As primary care delivery models of care continue to evolve, these findings may be useful in informing best practices for optimizing the delivery of preventive care services. 


\section{References}

1. Starfield B. Primary care : concept, evaluation, and policy. New York: Oxford University Press; 1992.

2. Institute of Medicine, National Academy of Sciences. A manpower policy for primary health care. Washington, D.C.: National Academies Press; 1978.

3. Jerant A, Fenton JJ, Franks P. Primary care attributes and mortality: a national person-level study. Ann Fam Med 2012;10:34-41.

4. American Academy of Family Physicians (AAFP), American Academy of Pediatrics (AAP), American College of Physicians (ACP), American Osteopathic Association (AOA). Joint principles of the patient-centered medical home. Patient-Centered Primary Care Collaborative, February 2007. Available from: http:// www.aafp.org/dam/AAFP/documents/practice_ management/pcmh/initiatives/PCMHJoint.pdf. Accessed September 24, 2015.

5. Jerant A, Bertakis KD, Fenton JJ, Franks P. Extended office hours and health care expenditures: a national study. Ann Fam Med 2012;10:388-95.

6. O'Malley AS. After-hours access to primary care practices linked with lower emergency department use and less unmet medical need. Health Aff (Millwood) 2013;32:175-83.

7. Starfield B, Shi L, Macinko J. Contribution of primary care to health systems and health. Milbank Q 2005;83:457-502.

8. Jaén CR, Ferrer RL, Miller WL, et al. Patient outcomes at 26 months in the patient-centered medical home National Demonstration Project. Ann Fam Med 2010;8(Suppl 1):S57-67.

9. Friedberg MW, Schneider EC, Rosenthal MB, Volpp KG, Werner RM. Association between participation in a multipayer medical home intervention and changes in quality, utilization, and costs of care. JAMA 2014;311:815-25.

10. Ferrante JM, Balasubramanian BA, Hudson SV, Crabtree BF. Principles of the patient-centered medical home and preventive services delivery. Ann Fam Med 2010;8:108-16.

11. Cogswell B, Eggert MS. People want doctors to give more preventive care. A qualitative study of health care consumers. Arch Fam Med 1993;2:611-9.

12. Assistant Secretary for Planning and Evaluation (ASPE). HHS Strategic Plan. Strategic Goal 1: Strengthen Health Care. Washington, DC: US Department of Health \& Human Services; 2015. Available from: http://www.hhs.gov/about/strategicplan/strategic-goal-1/index.html. Accessed September 24, 2015.

13. Stange KC, Kelly R, Chao J, et al. Physician agreement with US Preventive Services Task Force recommendations. J Fam Pract 1992;34:409-16.

14. Beal A, Hernandez S, Doty M. Latino access to the patient-centered medical home. J Gen Intern Med 2009;24(Suppl 3):514-20.
15. Bindman A, Grumbach K, Osmond D, Vranizan K, Stewart A. Primary care and receipt of preventive services. J Gen Intern Med 1996;11:269-76.

16. Flocke SA, Stange KC, Zyzanski SJ. The association of attributes of primary care with the delivery of clinical preventive services. Med Care 1998;36: AS21-30.

17. U.S. Preventive Services Task Force. Screening for breast cancer: U.S. Preventive Services Task Force recommendation statement. Ann Intern Med 2009; 151:716-26.

18. Colorectal cancer: screening. Recommendation summary. Rockville (MD): U.S. Preventive Services Task Force; 2008. Available from: http:// www.uspreventiveservicestaskforce.org/Page/ Topic/recommendation-summary/colorectalcancer-screening. Accessed September 24, 2015.

19. Cervical cancer: screening. Recommendation summary. Rockville (MD): U.S. Preventive Services Task Force; 2012. Available from: http://www. uspreventiveservicestaskforce.org/Page/Topic/ recommendation-summary/cervical-cancer-screening. Accessed September 24, 2015.

20. Lipid disorders in adults (cholesterol, dyslipidemia): screening. Recommendation summary. Rockville (MD): U.S. Preventive Services Task Force; 2008. Available from: http://www.uspreventiveservicestaskforce.org/Page/Topic/recommendation-summary/ lipid-disorders-in-adults-cholesterol-dyslipidemiascreening. Accessed September 24, 2015.

21. Centers for Disease Control and Prevention (CDC). Advisory Committee on Immunization Practices (ACIP) recommended immunization schedules for persons aged 0 through 18 years and adults aged 19 years and older-United States, 2013. MMWR Surveill Summ 2013;62(Suppl 1):1.

22. Prochazka AV, Lundahl K, Pearson W, Oboler SK, Anderson RJ. Support of evidence-based guidelines for the annual physical examination: a survey of primary care providers. Arch Intern Med 2005;165: 1347-52.

23. Voss JD, Schectman JM. Prostate cancer screening practices and beliefs. J Gen Intern Med 2001;16: 831-7.

24. Liu BY, O'Malley J, Mori M, et al. The association of type and number of chronic diseases with breast, cervical, and colorectal cancer screening. J Am Board Fam Med 2014;27:669-81.

25. Manuti B, Rizza P, Bianco A, Nobile CG, Pavia M. The quality of preventive health care delivered to adults: results from a cross-sectional study in Southern Italy. BMC Public Health 2010;10:350.

26. Thorpe JM, Kalinowski CT, Patterson ME, Sleath BL. Psychological distress as a barrier to preventive care in community-dwelling elderly in the United States. Med Care 2006;44:187-91. 
27. Fiscella K, Franks P, Clancy CM. Skepticism toward medical care and health care utilization. Med Care 1998;36:180-9.

28. Ware J Jr, Kosinski M, Keller SD. A 12-item ShortForm Health Survey: construction of scales and preliminary tests of reliability and validity. Med Care 1996;34:220-33.

29. Crabtree BF, Miller WL, Tallia AF, et al. Delivery of clinical preventive services in family medicine offices. Ann Fam Med 2005;3:430-5.

30. Jaen CR, Stange KC, Nutting PA. Competing demands of primary care: a model for the delivery of clinical preventive services. J Fam Pract 1994;38: 166-71.

31. Lewis CL, Couper MP, Levin CA, Pignone MP, Zikmund-Fisher BJ. Plans to stop cancer screening tests among adults who recently considered screening. J Gen Intern Med 2010;25:859-64.

32. Smith SK, Trevena L, Simpson JM, Barratt A, Nutbeam D, McCaffery KJ. A decision aid to support informed choices about bowel cancer screening among adults with low education: randomised controlled trial. BMJ 2010;341:c5370. 